ROSIANE MATTAR $^{1}$

MARIA REGINA TORLON $I^{2}$

Ana PILAR Betrán ${ }^{3}$

MARIO MERIALDI ${ }^{4}$

\title{
Obesidade e gravidez
}

\author{
Obesity and pregnancy
}

\section{O Problema}

Segundo a Organização Mundial da Saúde (OMS), sobrepeso e obesidade são definidos como acúmulo anormal ou excessivo de tecido adiposo que pode levar a prejuízos para a saúde ${ }^{1}$. A gestação pode atuar como desencadeante da obesidade, ou como agravante, quando aquela for pré-existente.

A obesidade é um grave problema de Saúde Pública. Sua prevalência vem aumentando sistematicamente ao longo das últimas décadas, tanto em países desenvolvidos como em boa parte dos países em desenvolvimento ${ }^{2}$. A situação mundial atual é tão grave que, no século 21, se fala em um epidemia global de obesidade - a chamada "globesidade" - que afetaria cinco dos seis continentes, poupando apenas a África Subsaariana. Segundo as últimas projeções da OMS, em 2005, existiriam pelo menos 400 milhões de adultos obesos em todo o mundo - um aumento de mais de 50\% em relação aos números de 1995; mais de um terço dessas pessoas habitam países em desenvolvimento ${ }^{1}$. Para facilitar a comparação da prevalência da obesidade entre diversos países, em 1996, a OMS criou uma base de dados sobre o índice de massa corpórea (IMC) de adultos de quase todos os países do mundo (por gênero e segundo faixa etária), que é atualizada periodicamente ${ }^{3}$. Ainda segundo a OMS, a obesidade seria hoje um dos maiores e mais visíveis, porém mais negligenciados, problemas de Saúde Pública em todo o mundo.

A epidemia de obesidade deve o seu grande avanço a uma série de fatores. Apesar de a predisposição genética ter papel relevante na suscetibilidade individual para se ganhar peso, o equilíbrio energético é basicamente resultante da ingestão calórica e da atividade física ${ }^{4}$. Aliado aos avanços dos meios de transporte e da disponibilidade de equipamentos que facilitam o desempenho de quase todas as atividades da vida diária, nunca o acesso à comida foi tão fácil, e os alimentos ricos em gordura e açúcar são geralmente os mais baratos. Portanto, os confortos da sociedade moderna criaram um ambiente "obesogênico".

Em 1994, Popkin ${ }^{5}$ chamou a atenção para o problema da "transição nutricional" dos países em desenvolvimento. Segundo esse autor, a troca de hábitos nutricionais tradicionais (baseados em grãos, fibras e frutas) por uma dieta ocidentalizada (rica em gordura saturada, alimentos refinados e açúcar) e um estilo de vida sedentário, além do aumento dos níveis de stress comuns ao meio urbano, resultariam em aumento das taxas de obesidade e doenças crônicas/degenerativas.

Departamento de Obstetrícia da Universidade Federal de São Paulo - UNIFESP - São Paulo (SP), Brasil; e Departamento de Saúde Reprodutiva e Pesquisa da Organização Mundial da Saúde - OMS - Genebra, Suíça.

'Professor associado da Universidade Federal de São Paulo - UNIFESP - São Paulo (SP), Brasil.

${ }_{2}^{2}$ Médica do Departamento de Obstetrícia da Universidade Federal de São Paulo - UNIFESP - São Paulo (SP), Brasil; e da Faculdade de Medicina do ABC - FMABC - Santo André (SP), Brasil.

${ }_{3}^{3}$ Médica da Unidade de Saúde Materna e Perinatal do Departamento de Saúde Reprodutiva e Pesquisa da Organização Mundial da Saúde - OMS - Genebra, Suíça.

${ }^{4}$ Coordenador da Unidade de Saúde Materna e Perinatal do Departamento de Saúde Reprodutiva e Pesquisa da Organização Mundial da Saúde - OMS - Genebra, Suiça. 
Como parte natural da epidemia mundial de obesidade, o número de mulheres em idade reprodutiva com sobrepeso também vem aumentando em todo o mundo ${ }^{4,6,7}$ - e o Brasil não é exceção. Dados do Instituto Brasileiro de Geografia e Estatística (IBGE) indicam que o excesso de peso e a obesidade entre as mulheres cresceram $50 \%$ nos últimos 30 anos, sendo que, atualmente, mais da metade $(51,9 \%)$ das brasileiras entre 20 e 44 anos estão com um IMC acima de $25^{8}$. Contrariamente ao que muitos acreditam, a prevalência de obesidade é maior entre as mulheres brasileiras de baixa renda do que nas classes sociais mais favorecidas ${ }^{8,9}$. Esse fenômeno não é exclusividade do Brasil e repete-se em quase todos os países em desenvolvimento com renda média intermediária ${ }^{2,10,11}$.

\section{Repercussões da obesidade sobre a concepção e a fertilidade}

Diversos estudos indicam que as obesas têm maior prevalência de amenorréia e infertilidade, sendo que 35 a $40 \%$ das mulheres com síndrome dos ovários policísticos são obesas ${ }^{12,13}$. A obesidade também reduziria as chances de sucesso dos tratamentos para esterilidade e aumentaria a probabilidade de abortamento espontâneo ${ }^{14,15}$.

\section{Repercussões maternas da obesidade}

Não existe um consenso quanto à definição de obesidade na gestação. Enquanto muitos autores utilizam como parâmetro o IMC $\left(\mathrm{kg} / \mathrm{m}^{2}\right)$ pré-gravídico ou no primeiro trimestre $>30$, outros utilizam o peso corporal da gestante superior a $150 \%$ do peso ideal ${ }^{16}$. Independente da definição, muitos estudos têm apontado que mulheres que iniciam a gravidez com IMC acima do normal $(20$ a 24,9) têm riscos mais elevados para diversas complicações. Por exemplo, segundo uma revisão sistemática, o risco de pré-eclâmpsia dobra a cada aumento de $5 \mathrm{a} 7 \mathrm{~kg} / \mathrm{m}^{2}$, o que equivale a um aumento no risco de 0,54\% (IC95\%=0,27-0,80) para cada $1 \mathrm{~kg} / \mathrm{m}^{2}$ de aumento do IMC. Essa relação se manteve mesmo nos estudos dos quais foram excluídas as mulheres com hipertensão crônica, diabetes ou gestação gemelar e após ajuste para possíveis fatores de confusão ${ }^{17}$; da mesma forma, quanto maior o IMC materno inicial, maior o risco de diabetes gestacional (DG). Segundo uma recente revisão sistemática que incluiu 70 estudos publicados nos últimos 40 anos, aquelas com sobrepeso (IMC $=25-29,9)$, quando comparadas às mulheres com IMC normal, teriam uma Odds Ratio (OR) para DG de 1,97 (IC95\%=1,77-2,19). Já mulheres com obesidade moderada (IMC=30-34,9) ou mórbida $($ IMC > 34,9) teriam OR de 3,10 (IC95\%=2,34-3,87) e 5,55 (IC95\%=4,27-7,21), respectivamente. Para cada aumento de $1 \mathrm{~kg} / \mathrm{m}^{2}$, a prevalência de DG aumentaria $0,92 \%(\mathrm{IC} 95 \%=0,73-1,10)^{18}$. As gestantes obesas também apresentam maior probabilidade de terem infecções urinárias e do trato genital inferior ${ }^{19}$. O sobrepeso materno aumenta ainda os riscos de parto induzido ${ }^{19}$, cesarianas $^{19,20}$, hemorragia maciça pós-parto e infecção puerperal ${ }^{19}$.

Comparadas às mulheres de peso normal, as obesas têm maior risco de morte não apenas na vida adulta, mas também no ciclo gravídico-puerperal, mesmo em países desenvolvidos. Segundo os dados mais recentes do relatório confidencial sobre saúde materna e infantil do Reino Unido, $35 \%$ de todos os casos de morte materna eram de mulheres obesas, comparado com $23 \%$ da população materna geral - um aumento dramático, se comparado aos $16 \%$ de obesidade no relatório de mortalidade de $1993^{21}$.

\section{Repercussões da obesidade sobre o concepto}

Os fetos são vítimas inocentes de diversos fatores ambientais adversos, entre eles os hábitos insalubres de suas mães. Enquanto os possíveis efeitos adversos do uso do álcool, fumo e drogas na gravidez são amplamente divulgados e conhecidos pela maioria dos médicos e leigos em geral, os riscos fetais decorrentes da obesidade materna são praticamente desconhecidos do grande público e também de muitos tocoginecologistas.

O excesso de tecido adiposo materno afetaria o concepto desde sua fase embrionária até o parto. A taxa de malformações fetais é maior em mulheres obesas do que naquelas com peso normal ${ }^{22-25}$. O excesso de tecido adiposo parece interferir no metabolismo dos folatos, o que explicaria a maior incidência de defeitos do tubo neural entre as obesas, mesmo naquelas que recebem suplementação de ácido fólico nas doses recomendadas ${ }^{22,24,26}$. O risco de óbito fetal é também significativamente maior entre as mulheres com peso acima da média, por motivos ainda desconhecidos ${ }^{27}$. A macrossomia fetal é mais frequente entre as obesas, independente da associação com diabetes ${ }^{16,28-30}$.

As obesas têm maior probabilidade de terem filhos obesos ${ }^{30-32}$, especialmente se elas tiverem também DG ou síndrome metabólica antes de iniciarem a gestação ou se houver ganho ponderal excessivo durante a gestação. O excesso de tecido adiposo materno parece ainda ser capaz de comprometer a programação metabólica fetal, predispondo os filhos de mulheres obesas a serem futuros obesos e diabéticos, perpetuando o ciclo da obesidade ${ }^{32-34}$. 


\section{Os desafios para a pesquisa e para a prática}

Ainda não conhecemos todos os mecanismos fisiopatológicos e quais são os mediadores envolvidos na associação entre obesidade materna e desfechos obstétricos adversos. Da mesma forma, ainda faltam estudos adequadamente desenhados e bem conduzidos (tipo coorte prospectivo multicêntrico) que avaliem a influência das características sociodemográficas sobre a associação obesidade/resultados obstétricos adversos. Por exemplo, será que a raça da paciente, seu país de origem ou sua paridade não modificariam a magnitude desse efeito? Talvez a pouca escolaridade, o baixo nível socioeconômico, a qualidade do pré-natal ou seu ganho ponderal durante a gestação também desempenhem um papel relevante na modulação dos riscos decorrentes da obesidade na gravidez. São também necessários estudos que investiguem o efeito de intervenções específicas durante o período pré-concepcional e no pré-natal para redução desses riscos. Por exemplo: qual é a efetividade de sessões educativas ou consultas periódicas com médicos, nutricionistas, enfermeiras ou fisioterapeutas para a redução do peso de mulheres obesas que planejam engravidar? Qual o seu impacto sobre o ganho ponderal de gestantes obesas?

A obesidade é uma doença altamente prevalente em nosso meio, que afeta todas as classes sociais - mas principalmente as mulheres de baixa renda. É uma doença preocupante, pois, além de expor suas portadoras a maiores riscos de doenças crônicas e degenerativas ao longo de suas vidas, também eleva os riscos de diversas complicações maternas e perinatais graves, inclusive de mortalidade.

É tempo de agir. O papel da OMS é oferecer diretrizes técnicas baseadas em evidências e disseminar exemplos de práticas eficazes, incentivando o envolvimento de todos os segmentos da sociedade na luta contra a obesidade. Em 2004, foi lançada uma estratégia mundial que oferece orientações práticas e claras sobre formas de agir, com sugestões de implementação, avaliação e monitoramento contínuos ${ }^{35}$.

As organizações profissionais e de classe devem apoiar os esforços das autoridades governamentais na prevenção e no tratamento da obesidade e suas complicações. Os consumidores, os leigos e suas organizações podem colaborar divulgando informações e mantendo o público em geral ciente dos riscos decorrentes da obesidade. Os profissionais especializados na saúde da mulher devem se aliar às autoridades governamentais para estabelecer metas, planejar $\mathrm{e}$ implementar estratégias de prevenção primária, de tratamento e de controle da obesidade para suas pacientes em todas as fases de suas vidas. Durante a gestação, é preciso estar atento e conhecer os riscos específicos associados ao binômio obesidade/gravidez, e implementar as melhores intervenções existentes para tentar reduzir esses riscos, além de educar e encorajar as pacientes para que planejem engravidar com um peso corporal adequado na próxima gestação. Afinal, assim como avisamos com naturalidade nossas gestantes que fumar acarreta riscos para sua saúde e a do seu bebê, deveríamos também informá-las, de forma clara e objetiva, sobre os riscos decorrentes do excesso de peso.

\section{Referências}

1. World Health Organization. Division of Noncommunicable Diseases. Programme of Nutrition Family and Reproductive Health. Obesity: preventing and managing the global epidemic: report of a WHO consultation on obesity. Geneva: WHO; 1998.

2. Martorell R, Khan LK, Hughes ML, Grummer-Strawn LM. Obesity in women from developing countries. Eur J Clin Nutr. 2000;54(3):247-52.

3. World Health Organization. Global Database on Body Mass Index: an interactive surveillance tool for monitoring nutrition transition [Internet]. Geneva: WHO; 2009 [cited 2009 Jan 21]. Available from: <http://www.who.int/bmi/index.jsp>

4. World Health Organization. Global strategy on diet, physical activity and health: obesity and overweight [Internet]. Geneva: WHO; 2003 [cited 2009 Jan 21]. Available from: <http://www.who.int/hpr/NPH/docs/gs_obesity.pdf>

5. Popkin BM. The nutrition transition in low-income countries: an emerging crisis. Nutr Rev. 1994;52(9):285-98.

6. Pleis JR, Lethbridge-Cejku M. Summary health statistics for U.S. adults: National Health Interview Survey, 2006. Vital Health Stat 10. 2007;(235):1-153.

7. Mansour D. Implications of the growing obesity epidemic on contraception and reproductive health. J Fam Plann Reprod Health Care. 2004;30(4):209-11.

8. Instituto Brasileiro de Geografia e Estatística. Pesquisa de Orçamentos Familiares - POF 2002-2003 [Internet]. Rio de Janeiro: IBGE; 2004 [citado 2009 Jan 20]. Disponível em: http://www.ibge.gov.br/home/estatistica/populacao/condicao devida/pof/2002/pof2002.pdf

9. Monteiro CA, Moura EC, Conde WL, Popkin BM. Socioeconomic status and obesity in adult populations of developing countries: a review. Bull World Health Organ. 2004;82(12):940-6. 
10. Monteiro CA, Conde WL, Lu B, Popkin BM. Obesity and inequities in health in the developing world. Int J Obes Relat Metab Disord. $2004 ; 28(9): 1181-6$.

11. Monteiro CA, Conde WL, Popkin BM. The burden of disease from undernutrition and overnutrition in countries undergoing rapid nutrition transition: a view from Brazil. Am J Public Health. 2004;94(3):433-4.

12. Yu CK, Teoh TG, Robinson S. Obesity in pregnancy. BJOG. 2006;113(10):1117-25.

13. Hart R, Hickey M, Franks S. Definitions, prevalence and symptoms of polycystic ovaries and polycystic ovary syndrome. Best Pract Res Clin Obstet Gynaecol. 2004; 18(5):671-83.

14 Bellver J, Rossal LP, Bosch E, Zúñiga A, Corona JT, Meléndez F, et al. Obesity and the risk of spontaneous abortion after oocyte donation. Fertil Steril. 2003;79(5):1 136-40.

15. Fedorcsak P, Storeng R, Dale PO, Tanbo T, Abyholm T. Obesity is a risk factor for early pregnancy loss after IVF or ICSI. Acta Obstet Gynecol Scand. 2000;79(1):43-8.

16. Isaacs JD, Magann EF, Martin RW, Chauhan SP, Morrison JC. Obstetric challenges of massive obesity complicating pregnancy. J Perinatol. $1994 ; 14(1): 10-4$.

17. O'Brien TE, Ray JG, Chan WS. Maternal body mass index and the risk of preeclampsia: a systematic overview. Epidemiology. $2003 ; 14(3): 368-74$.

18. Torloni MR, Betrán AP, Horta BL, Nakamura MU, Atallah AN, Moron AF, et al. Prepregnancy BMl and the risk of gestational diabetes: a systematic review of the literature with meta-analysis. Obes Rev. 2009;10(2):194-203.

19. Sebire NJ, Jolly M, Harris JP, Wadsworth J, Joffe M, Beard RW, et al. Maternal obesity and pregnancy outcome: a study of 287,213 pregnancies in London. Int J Obes Relat Metab Disord. $2001 ; 25(8): 1175-82$.

20. Chu SY, Kim SY, Schmid CH, Dietz PM, Callaghan WM, Lau J, et al. Maternal obesity and risk of cesarean delivery: a meta-analysis. Obes Rev. 2007;8(5):385-94.

21. Lewis G, editor. Why mothers die 2000-2002: the sixth report of the confidential enquires into maternal deaths in the United Kingdom. London: CEMACH; 2004.

22. Anderson JL, Waller DK, Canfield MA, Shaw GM, Watkins ML, Werler MM. Maternal obesity, gestational diabetes, and central nervous system birth defects. Epidemiology. 2005;16(1):87-92.

23. Martínez-Frías ML, Frías JP, Bermejo E, Rodríguez-Pinilla E, Prieto L, Frías JL. Pre-gestational maternal body mass index predicts an increased risk of congenital malformations in infants of mothers with gestational diabetes. Diabet Med. 2005;22(6):775-81.

24. Rasmussen SA, Chu SY, Kim SY, Schmid CH, Lau J. Maternal obesity and risk of neural tube defects: a metaanalysis. Am J Obstet Gynecol. $2008 ; 198(6): 611-9$.

25. Ray JG, Vermeulen M, Meier C, Wyatt PR. Risk of congenital anomalies detected during antenatal serum screening in women with pregestational diabetes. QJM. 2004;97(10):651-3.

26. Ray JG, Wyatt PR, Vermeulen M, Meier C, Cole DE. Greater maternal weight and the ongoing risk of neural tube defects after folic acid flour fortification. Obstet Gynecol. 2005;105(2):261-5.

27. Chu SY, Kim SY, Lau J, Schmid CH, Dietz PM, Callaghan WM, et al. Maternal obesity and risk of stillbirth: a metaanalysis. Am J Obstet Gynecol. 2007; 197(3):223-8.

28. Cnattingius S, Bergstrom R, Lipworth L, Kramer MS. Prepregnancy weight and the risk of adverse pregnancy outcomes. N Engl J Med. 1998;338(3): 147-52.

29. Jensen DM, Damm P, Sørensen B, Mølsted-Pedersen L, Westergaard JG, Ovesen P, et al. Pregnancy outcome and prepregnancy body mass index in 2459 glucose-tolerant Danish women. Am J Obstet Gynecol. 2003; 189(1):239-44.

30. Kramer MS, Morin I, Yang H, Platt RW, Usher R, McNamara H, et al. Why are babies getting bigger? Temporal trends in fetal growth and its determinants. J Pediatr. 2002;141(4):538-42.

31. Surkan PJ, Hsieh CC, Johansson AL, Dickman PW, Cnattingius S. Reasons for increasing trends in large for gestational age births. Obstet Gynecol. 2004; 104(4):720-6.

32. Whitaker RC. Predicting preschooler obesity at birth: the role of maternal obesity in early pregnancy. Pediatrics. 2004;1 14(1):e29-36.

33. Johannsson E, Arngrimsson SA, Thorsdottir I, Sveinsson T. Tracking of overweight from early childhood to adolescence in cohorts born 1988 and 1994: overweight in a high birth weight population. Int J Obes (Lond). 2006;30(8):1265-71.

34. Siega-Riz AM, Siega-Riz AM, Laraia B. The implications of maternal overweight and obesity on the course of pregnancy and birth outcomes. Matern Child Health J. 2006; 10 (5 Suppl):S153-6.

35. World Health Organization. Global strategy on diet, physical activity and health: a framework to monitor and evaluate implementation [Internet]. Geneva: WHO; 2008 [cited 2009 Jan 21 ]. Available from: <http://www.who.int/dietphysicalactivity/DPASindicators/en/index.html> 